\title{
Psicologia da Saúde no Brasil ${ }^{1}$
}

\author{
Ricardo Gorayeb ${ }^{2}$ \\ Universidade de São Paulo
}

\begin{abstract}
RESUMO - O artigo apresenta uma descrição do desenvolvimento da Psicologia da Saúde no Brasil a partir do ponto de vista do autor, com o relato de suas próprias experiências, desde sua graduação até hoje. São enfatizadas suas experiências de aprendizagem com professores relevantes para o desenvolvimento da Psicologia e da Psicologia da Saúde no país. Alguns termos, utilizados de maneira confusa, como Psicologia da Saúde x Psicologia Clínica x Psicologia Hospitalar são analisados. A produção científica da área é avaliada, bem como discutidas formas de incremento. Efetua-se também uma análise de instrumentos necessários ao desenvolvimento da área, sendo identificados alguns grupos de pesquisadores em Psicologia da Saúde com base em evidências e apontada uma perspectiva de crescimento da área.
\end{abstract}

Palavras-chave: Psicologia e Saúde, Brasil, História.

\section{Health Psychology in Brazil}

\begin{abstract}
This article presents a description of the development of Health Psychology in Brazil from the author's viewpoint, reporting his experiences in the area, since his undergraduate studies until nowadays. The author's learning experiences with professors that were relevant to the development of Psychology and Health Psychology in the country are emphasized. Some misunderstood terms, such as Health Psychology x Clinical Psychology x Psychology in Hospitals are analyzed. This paper also evaluates the scientific production in the area, as well as ways to increase such production. Finally, an analysis of the instruments needed to develop the area is performed, pointing out some research groups working in evidence-based Health Psychology in Brazil, and presenting a perspective of growth of the area.
\end{abstract}

Keywords: Health Psychology, Brazil, History.

Honrado com o convite para participar desta edição de celebração dos 25 anos de publicação ininterrupta da revista Psicologia: Teoria e Pesquisa, escrevendo um artigo sobre o tema Psicologia da Saúde no Brasil, junto-me aos demais autores no cumprimento aos editores atuais e seus predecessores, pelo empenho e dedicação à divulgação do conhecimento científico no Brasil. Por seu trabalho, a revista Psicologia: Teoria e Pesquisa adquiriu, ao longo desses anos, um nível de respeito e competência invejáveis e desejáveis a qualquer periódico científico.

Ao aceitar o convite, deparei-me, ao mesmo tempo, com a enorme responsabilidade de ser o autor do único artigo que vai tratar do tema neste número especial e com os limites impostos pela característica da publicação, a ser executada em tempo curto para tarefa tão relevante. Todavia, aceitei, e tomo a liberdade de fazê-lo de um modo muito pessoal, descrevendo um pouco de minhas percepções e minha própria história na área, mesmo correndo o risco de ser injusto ou impreciso ao tratar dos aspectos históricos mais gerais envolvidos e, especialmente, com a possibilidade de omissão de nomes de pesquisadores ou professores que foram relevantes, mas que não fizeram parte da história vista sob minha perspectiva ou que não foram abrangidos

1 Agradeço à colaboração da psicóloga Ana Luisa Suguihura no levantamento bibliográfico para a elaboração deste artigo.

2 Endereço para correspondência: Av. Bandeirantes, 3.900. Ribeirão Preto, SP. CEP 14048-900. E-mail: rgorayeb@fmrp.usp.br por minhas investigações sobre o tema. Um limite importante que vou estabelecer neste texto é o fato que vou falar de Psicologia da Saúde com base em evidências, por ser a maneira que mais respeito para se tratar um problema científico. Assim, autores ou publicações brasileiros que não abordaram a saúde com base em evidências científicas não serão contemplados no texto. Aos outros que assim o fizeram, mas que, por omissão minha, não são citados no texto, minhas apologias. A ciência se constrói gradualmente, assim, outros artigos já escritos (e.g., Kerbauy, 2002; Pires \& Braga, 2009; Sebastiani, 2003) ou que serão escritos posteriormente a este, poderão sempre complementá-lo e criticá-lo, ajudando na construção de uma história mais representativa de todos os fatos. Deixo assim aqui minha impressão sobre o desenvolvimento da Psicologia da Saúde no Brasil, enfatizando que esta é uma visão particular, não tenta descrever detalhada e exaustivamente a história total e completa, mas é sim um ângulo da história, com os vieses característicos a uma abordagem peculiar.

O tema Psicologia da Saúde me é caro e fico feliz por poder contribuir para sua divulgação no nível de excelência desta publicação. Venho trabalhando na questão há 40 anos, desde que me tornei psicólogo, em 1970, pela Faculdade de Filosofia, Ciências e Letras de Ribeirão Preto, então um instituto isolado da Secretaria de Educação do Estado de São Paulo, hoje USP (FFCLRPUSP). Desde aquela época sofri a influência de diversos Mestres, a quem vou reverenciar, com prazer, neste artigo. Essas influências me tornaram o 
Psicólogo da Saúde que sou e moldaram a maneira de formar os alunos que formei.

Já anteriormente aos tempos de faculdade, fruto de minha educação familiar, na infância e adolescência sempre fui instado a procurar ajudar ao próximo, fazer sempre o melhor, comprovar o que fazia ou dizia, buscar a excelência nas minhas ações pessoais. Isso certamente me influenciou a optar por uma abordagem científica que utilizasse relações causais e direcionou minha formação acadêmica, dentre as diversas abordagens psicológicas, para uma Psicologia baseada em evidências. Essa influência para buscar o melhor, procurar sempre acertar e ter uma visão humanista do mundo, concretizou-se na minha formação profissional, inicialmente no curso de graduação em Psicologia. Enquanto ainda aluno de primeiro ano, em 1966, fui orientado nos primeiros passos dentro da Análise do Comportamento pelo Prof. Luiz Marcellino de Oliveira (ver Bueno, 2006; Ferrari, 2008), o qual era instrutor da disciplina Teorias de Aprendizagem. Luiz sofrera direta e recentemente uma influência da Universidade de Brasília de antes do golpe militar, onde tinha sido aluno da Profa. Carolina Bori (ver o volume 9, número 1, de 1998, da revista Psicologia USP, que é todo dedicado a ela; Feitosa, 2007; Gorayeb, 2005; Guedes, 2005; Kerbauy, 2004) e do Prof. Fred S. Keller (além de sua extensa obra, ver Cook, 1996; Matos, 1998). De Keller, Luiz trazia o modelo de Ensino Programado Individualizado (Keller, 1966), que aplicava em seu curso. Fui convidado por Luiz, em 1966, junto com outros alunos que se sobressaíram na primeira versão do curso, a atuar como monitor para a turma seguinte. Essa foi minha primeira experiência como "professor", atendendo ao princípio que Keller muito enfatizava, que era a proximidade entre instrutor e aprendiz, para facilitar a aprendizagem. Junto com outras características do método, como o uso de passos graduais de aprendizagem, o respeito ao ritmo de cada aluno, o domínio de $100 \%$ do conteúdo antes de ser promovido ao passo seguinte, fui tendo contato real com os princípios da Análise do Comportamento, aplicados ao meu próprio comportamento de aprender e ensinar. Ver o respeito ao aluno no processo de aprendizagem começou a exercer sua influência sobre minha maneira de ver o sujeito, o indivíduo e, posteriormente, influiu também na minha percepção do que poderia ou deveria ser oferecido ao paciente. Gosto de parafrasear Keller (1968), que dizia "o aluno tem sempre razão", ao afirmar, orgulhoso, que comecei a aprender com o Sistema Personalizado de Instrução, não só a concreta aplicação desta máxima nas atividades educacionais, mas também sua generalização para a prática em Psicologia da Saúde. Ao dizer e ensinar que "o paciente tem sempre razão!", em ações com respeito à sua individualidade e com uma visão crítica constante dos procedimentos que utilizamos, busco o aperfeiçoamento das intervenções e maior eficiência, tal como Keller ensinou.

Meu primeiro contato com a Psicologia da Saúde ocorreu enquanto ainda era estudante de graduação, atendendo convite feito pela Profa. Maria Clotilde Rossetti Ferreira (ver Dessen, 2006) para ser estagiário de uma professora da Faculdade de Medicina de Ribeirão Preto (FMRPUSP), sua amiga, Thereza Pontual de Lemos $\mathrm{Mettel}^{3}$, com quem os alunos de então da FFCLRPUSP ainda não tinham tido contato.

Thereza Mettel foi uma das primeiras psicólogas brasileiras a atuar dentro da área da saúde, mais especificamente dentro do Hospital das Clínicas da Faculdade de Medicina de Ribeirão Preto da Universidade de São Paulo (HCFMPUSP). Thereza foi, para mim, bem como para outros professores, pesquisadores e psicólogos clínicos, uma tutora altamente qualificada, gentil, eficiente, constituindo-se, assim, num modelo de atuação pessoal e profissional. Iniciei com Thereza meu primeiro estágio clínico, no princípio apenas observando e registrando comportamentos de pacientes e terapeutas, em uma sala com espelho unidirecional, onde se realizava o atendimento clínico que ela e outros estagiários mais graduados que eu, como Célia Maria Lana da Costa Zannon, faziam com pacientes. Gradualmente, com o mais adequado uso das técnicas de aproximação sucessiva e reforço diferencial, Thereza foi me introduzindo às responsabilidades do atendimento integral de um paciente, desde o planejamento antecipado das sessões, a consideração dos aspectos éticos no relacionamento, a valorização e importância da adequada comunicação com os outros profissionais, até o seguimento após a alta, numa avaliação do progresso do paciente e da eficácia da intervenção. Não fazíamos pesquisa, mas a sistematização que Thereza aplicava a seu trabalho, os cuidados e detalhes na análise dos resultados de cada intervenção, colaboraram para moldar em mim as características de pesquisador clínico. Fui seu estagiário por três anos, de 1968 a 1970, cumprindo mais de 1.500 horas de estágio, nos últimos anos de minha graduação, num treino muito diferenciado e qualificado para tornar-me um terapeuta comportamental.

Ainda durante o curso de graduação, por influência da Profa. Ângela Rozestraten, responsável pela disciplina de ética na FFCLRPUSP, tive a oportunidade de atuar ativamente, junto com um grupo de alunos e professores desta faculdade e da FMRPUSP, na criação, em 1970, da Sociedade de Psicologia de Ribeirão Preto (SPRP). A SPRP, pela relevância de sua atuação no cenário científico nacional, viria posteriormente a transformar-se na Sociedade Brasileira de Psicologia - SBP (Gorayeb, 1990; Rozestraten, 1988). O papel das sociedades científicas será abordado mais tarde neste artigo, mas gostaria já de ressaltar que a SPRP, construída a partir da somatória de esforços de estudantes e professores, visando somente o desenvolvimento da ciência e a criação de um espaço de interação, sem privilégios para atitudes personalistas ou ego centradas, é a meu ver o modelo ideal para o funcionamento das sociedades científicas. A existência da SPRP e da SBP foi muito importante para os primeiros passos da Psicologia no Brasil, tendo um espaço aberto em seus congressos e na antiga "Divisão de Modificação de Comportamento" para divulgação de conhecimento científico, troca de experiências e formação profissional na incipiente área de Psicologia da Saúde.

3 Por ocasião de sua aposentadoria, foi justamente homenageada pela Universidade de Brasília com o título de Professor Emérito (ver texto produzido pelos professores do Departamento de Psicologia Escolar e do Desenvolvimento/UnB, 2007). 
No ambiente do antigo Departamento de Neurologia, Psiquiatria e Psicologia Médica, hoje Departamento de Neurociências e Ciências do Comportamento da FMRPUSP, conheci também o Prof. João Cláudio Todorov ${ }^{4}$. Apesar de eu estar vivamente interessado na área clínica, Thereza não dispunha, quando me formei, de bolsas para me receber como aluno de mestrado. João Cláudio tinha uma vaga de bolsista e convidou-me a cursar o Programa de Mestrado em Psicologia Experimental no Instituto de Psicologia da USP, em São Paulo, sob sua orientação, levando-me de volta à pesquisa básica com animais, onde eu já tivera minha "iniciação" como monitor de alunos de graduação. Nesse laboratório, tive o prazer de conviver com a Profa. Deisy das Graças de Souza, na época estagiária de graduação, hoje professora e pesquisadora renomada em Análise do Comportamento, e também com o então cirurgião dentista Prof. Antonio Bento Alves de Moraes, hoje importante pesquisador na área de Odontologia Comportamental. Eles eram também orientandos de João Cláudio, que era o líder científico na área de Psicologia naquele momento em Ribeirão Preto. Desenvolver pesquisas sob sua orientação fortaleceu em mim, e acredito que em todos seus alunos, a importância da atividade sistemática e consistente para a produção de dados confiáveis e do adequado respaldo teórico por detrás da coleta de dados. Pelo fato de não existir um laboratório específico na FMRPUSP para que João Cláudio pudesse desenvolver suas pesquisas em Análise do Comportamento, estas ocorriam no Laboratório de Psicofarmacologia do Prof. Frederico Graeff (ver Jurberg, 2010). Mesmo não vindo mais a trabalhar em pesquisa básica após o doutorado, o convívio diário com esses dois grandes nomes da pesquisa em Análise do Comportamento e em Psicofarmacologia exerceu grande influência em minhas atitudes posteriores como investigador em Psicologia da Saúde.

Com a mudança para melhor do clima político na UnB, João Cláudio e Thereza foram convidados a trabalhar naquela Universidade. Com a saída de Thereza da FMRPUSP, fui contratado, em 1974, para ocupar sua vaga, no departamento onde era aprendiz. Thereza havia iniciado um processo de atenção aos pacientes dentro do hospital, e a mim cabia dar continuidade a ele. Escassos eram os recursos, não havia pessoal contratado para apoio e os únicos psicólogos eram os próprios docentes. Assim, com a colaboração da colega de departamento, Profa. Edna Maria Marturano, iniciou-se no HCFMRPUSP a primeira Residência em Psicologia do Brasil, utilizando a mesma estrutura e modelo de atuação da Residência Médica, com o estratégico apoio do presidente da Comissão de Residência Médica do Hospital, o neurologista José Geraldo Speciali. Tratava-se de um programa de treinamento em serviço, onde o psicólogo recém-formado permanecia em tempo integral no hospital, realizando atendimentos nos ambulatórios ou nas enfermarias. Descrições do Programa de Residência e das primeiras incursões em Psicologia da Saúde no HCFMRPUSP podem ser vistas em Gorayeb (1983, 1984, 1985). Esse Programa já formou até

4 Também foi homenageado pela Universidade de Brasília com o título de Professor Emérito (ver Cunha, 2007 e o texto produzido pelos professores do Departamento de Processos Psicológicos Básicos/UnB, 2007). hoje mais de uma centena de residentes, incluindo, a título de exemplo, psicólogas que depois vieram a se tornar destaques em Psicologia e Saúde, como Maria Aparecida Crepaldi e Maria Beatriz Martins Linhares.

Esse modelo de residência acabou servindo para que o Conselho Federal de Psicologia criasse normas para a regulamentação dos programas de Residência em Psicologia no país (Resolução CFP No 015/2007). O programa pioneiro criado naquela época existe até hoje, com o inadequado nome de "Programa de Aprimoramento", sendo o HCFMRPUSP um dos centros de referência na área de Psicologia da Saúde no Brasil. Essa mudança de nome foi uma atitude "estratégica" da FUNDAP e da Secretaria da Saúde do Estado de São Paulo, que pagavam as bolsas dos aprendizes, para diferenciar os programas de "aprimoramento" dos programas de "residência", vindo, assim, a reduzir os valores da bolsa dos chamados "aprimorandos" para valores até cinco vezes menores que aqueles pagos aos residentes médicos. Recentemente, o Ministério da Saúde e o Ministério da Educação, em suas próprias palavras, como "resultado de ampla articulação e intenso trabalho junto às diversas entidades e instituições representativas da área da saúde desde 2005", vieram a atuar no sentido de ajudar a corrigir a escassez de programas de formação de outros profissionais de saúde, que não o médico, criando o Programa de Residência Multiprofissional em Saúde e em Área Profissional da Saúde. Nesse novo programa, os valores da bolsa são os mesmo que para os residentes médicos, dando início a um processo de correção da discriminação que existe de longa data contra profissionais de saúde não médicos, para, quiçá no futuro, eliminá-la. Para sua implantação, foi publicada, em 2007, a Portaria Interministerial $n^{\circ} 45$, que dispõe sobre a Residência Multiprofissional em Saúde e a Residência em Área Profissional da Saúde, sendo posteriormente instituída uma Comissão Nacional de Residência Multiprofissional em Saúde (CNRMS), por meio da Portaria Interministerial MEC/ MS n 698 (Ministério da Educação, 2007).

Mesmo nas fases iniciais do desenvolvimento do Serviço de Psicologia do HCFMRPUSP e da Residência em Psicologia, dado o ineditismo de tais atuações no país, ali recebemos visitas que tinham o intuito de observar o modelo de trabalho de um Serviço de Psicologia dentro de um hospital geral e de formação pós-graduada de psicólogos na área da Saúde. Visitaram-nos, para conhecer nossa maneira de trabalhar, professoras que hoje são destaque em Psicologia da Saúde, como a Profa. Maria Cristina Miyazaki, da Faculdade de Medicina de São José do Rio Preto (FAMERP), e a Profa. Belkiss Romano, do Instituto do Coração da Faculdade de Medicina da USP.

Voltei a viajar semanalmente para São Paulo, em 1978, para cursar o Programa de Doutorado em Psicologia Experimental, no Bloco 10 da USP, agora sob a orientação de Carolina Bori. Quase desnecessário dizer que, com o privilégio de ter Carolina como orientadora e convivendo com outros colegas e professores, nesse ambiente só se "respirava" o clima de uma Psicologia com base em evidências. Carolina foi uma orientadora exemplar, no sentido de estimular minha independência no processo de coleta de dados e de redação científica. Com ela também tive estimulados meus comportamentos de cuidado com o outro, atenção para as políticas 
nacionais na área das ciências, em especial a psicológica, e a valorização da importância das sociedades científicas. No programa de doutorado, meu processo de formação foi complementado com aulas com docentes do calibre da Profa. Maria Amélia Matos (ver Assis, Delitti \& Lima, 2005; Tomanari, 2005) e da Profa. Dora Fix Ventura, entre outros grandes nomes.

Fred Keller de vez em quando se fazia presente e se tornava, cada vez mais, um ícone desse processo de formação em Psicologia fundamentada na Ciência. Tive a oportunidade de interagir com ele em algumas ocasiões, em Ribeirão Preto e em São Paulo. Sua presença sempre causava um grande impacto positivo entre os alunos. Posteriormente, vim a conhecer mais intimamente essa figura humana incomparável dentro da área de Análise do Comportamento, quando fiz meu pós-doutorado na Duke University, residindo em Chapel Hill, sua cidade, na Carolina do Norte, EUA, e tendo a oportunidade ímpar de conviver com ele e D. Frances, sua esposa e companheira, em sua casa. Uma das características de Keller era que seus ensinamentos sempre ultrapassavam o limite da sala de aula ou de qualquer outro contexto de aplicação profissional, em geral servindo como modelo de vida e de trabalho. Seu papel na difusão da Análise do Comportamento é, a meu ver, mais relevante que o papel do próprio B. F. Skinner (1904-1990).

Bem, conto todas essas história porque um cientista ou um psicólogo não nasce do nada, não é fruto só de sua genética, de seu próprio cérebro, de sua educação formal, ou dos livros que lê, mas sim, e principalmente, é o resultado das influências que recebeu de seus mestres. E, tenho aqui, neste artigo de realce, que dar o devido destaque aos professores que me formaram. Como disse o próprio Isaac Newton (16431727), "se enxerguei mais longe, foi porque estava apoiado sobre os ombros de gigantes"; assim, também ouso dizer que se construí algo em Psicologia da Saúde no Brasil foi porque também estava apoiado sobre os ombros de gigantes da Psicologia brasileira.

Além dos pesquisadores e professores que tiveram influência direta sobre minha formação, é necessário também ressaltar a importância da presença de dois outros professores estrangeiros que estiveram por um período relativamente longo no Brasil e contribuíram para o desenvolvimento da Análise do Comportamento Aplicada e, consequentemente, para a Psicologia da Saúde. Fred Keller foi, sem dúvida, o professor estrangeiro que mais contribuiu para esse desenvolvimento, mas alguns professores mais jovens também tiveram seu papel. A presença, em São Carlos, de Larry William, atuando no Programa de Pós-Graduação em Educação Especial da Universidade Federal de São Carlos (UFSCar), influiu na formação de profissionais bem qualificados nessa área. A presença, no Rio de Janeiro, de Harald Lettner e sua associação com Bernard Rangé influíram na produção de livros, com destaque para o primeiro (Lettner \& Rangé, 1988) do conjunto que Bernard continua a editar na área de Psicologia Clínica. Esses professores tiveram, e seus legados têm, até hoje, impacto na área da Saúde. Sua presença também colaborou para a organização da Associação Brasileira de Psicoterapia e Medicina Comportamental (ABPMC), fundada em 1991 e hoje dirigida pela Profa. Martha Hubner.
Além dos professores já citados, cumpre destacar o papel pioneiro de outros psicólogos brasileiros na área de Psicologia da Saúde. Primeiro, tenho que citar a Profa. Mathilde Neder, do Hospital das Clínicas da Faculdade de Medicina da Universidade de São Paulo, certamente uma pioneira na área (ver exemplo de suas publicações em Neder, 1995; ver também a homenagem que lhe foi prestada pelo Conselho Federal de Psicologia, 2005). O nome da Profa. Raquel Rodrigues Kerbauy, do Instituto de Psicologia da USP, é um que certamente não pode deixar de ser citado, por sua contribuição como professora, por suas publicações (e. g., Kerbauy, 1999) e por sua atuação na área da saúde na Associação Nacional de Pesquisa e Pós Graduação em Psicologia (ANPEPP). Além dela, destaco a Profa. Vera Raposo do Amaral, da PUC de Campinas e do Hospital da Sociedade de Pesquisa e Assistência para Reabilitação Craniofacial (SOBRAPAR), que também trilhou os primeiros caminhos da Análise do Comportamento aplicada à situação de saúde, trazendo contribuições importantes e ajudando a formar muitos psicólogos que partiram para atuar em diversos outros hospitais do Brasil. Também, já há vários anos, a Profa. Silvia Cury Ismael, do Hospital do Coração em São Paulo, implantou um Serviço de Psicologia com base em evidências em uma instituição privada/filantrópica, e mantém até hoje uma base de investigação em suas ações. Além dessas pessoas, muitas outras têm trazido, nos últimos 10 ou 20 anos, importantes contribuições para a sistematização do conhecimento na área da saúde, seja em ambientes hospitalares seja em outros ambientes, como Fani Malerbi, na PUCSP; Neide Micelli e Nelson Valério, na Faculdade de Medicina de São José do Rio Preto (FAMERP); Maria Rita Zoega Soares, na Universidade Estadual de Londrina; Ricardo Sebastiani e Sheila Murta, na PUC de Goiânia. Muitos outros nomes podem ser acrescidos a essa lista, mas este não é propriamente o objetivo deste artigo, conforme salientado nos parágrafos iniciais.

O certo é que a produção brasileira na área de Psicologia da Saúde tem crescido muito nos últimos anos, mesmo não tendo ainda atingido uma diversidade de temas e uma quantidade de artigos significativos para a formação adequada de psicólogos da saúde necessários ao país, ou para a ampla disseminação de conhecimentos. Como exemplo de um crescimento ainda insuficiente, nota-se que as primeiras publicações no Brasil, na base de dados LILACS, usando os descritores "Psicologia", "Saúde" e "Brasil", somente surgiram em 1982, sendo que no período 1982-1989 apenas 40 artigos foram publicados. Na década de 1990, observa-se um crescimento de $250 \%$ e, na última década (de 2000 a 2009), outro crescimento de mais de $300 \%$ sobre a década anterior. Esses dados a princípio poderiam indicar um grande aumento da produção, mas isso precisa ser relativizado. Vejamos, por exemplo, que a produção encontrada no MEDLINE, usando os descritores "Psychology", "Health" $e$ "Brazil", mostra que ali os primeiros textos surgiram em 1971, 11 anos antes que os primeiros textos na base de dados LILACS, e que a década de 1990 teve um crescimento de $140 \%$ sobre a década anterior, e a década seguinte (2000 a 2009) teve um crescimento de $180 \%$ sobre a década precedente. Assim, nota-se que a produção descrita na base de dados MEDLINE é 3,5 vezes menor que a descrita na base de dados LILACS. Apesar de a 
produção descrita em ambas as bases de dados refletir um aumento no ritmo de atividade científica na área, se comparada com a de outras áreas da Psicologia, essa produção ainda é pequena. Os psicólogos da saúde brasileiros ainda publicam pouco, especialmente em revistas internacionais, especialmente as indexadas e com bom índice de impacto. $\mathrm{O}$ que se tem observado ultimamente é que a pressão das universidades, a seletividade das agências de fomento, a maior interação dos centros de pós-graduação do Brasil entre si e com outros centros no exterior, podem aumentar o número de publicações e qualificar melhor a produção científica no futuro.

Esses dados brevemente analisados se referem à publicação em revistas periódicas, mas também se nota um aumento na produção de livros em Psicologia da Saúde. Alguns livros traduzidos são importantes para a área, como o de Straub (2005). Outros livros nacionais - teóricos ou com relatos detalhados de experiências bem sucedidas em Psicologia da Saúde - são úteis para criar modelos para uma atuação adequada e indicativos de uma produção crescente na área. Como exemplo, temos os livros de Kerbauy (1999), Ismael (2005), Miyazaki, Domingos e Valério (2006) e Crepaldi, Linhares e Perosa (2006). Novamente, não se pretende ser exaustivo. Além desses, dois livros estão previstos para publicação num futuro próximo: um livro sobre Psicologia da Saúde, pelo Grupo de Trabalho "Psicologia da Saúde em Instituições e na Comunidade" da ANPEPP, e um livro em Psicologia da Saúde no Ambiente Hospitalar, descrevendo as experiências do Serviço de Psicologia do HCFMRPUSP, editado sob minha coordenação. As publicações na área de Psicologia da Saúde têm enorme capacidade de aumento quantitativo e qualitativo e enfatiza-se aqui a necessidade de produção de mais livros texto e artigos em periódicos que possam ser utilizados na formação de novos profissionais que ingressam na área.

\section{O Uso de Termo Psicologia da Saúde}

O termo Psicologia da Saúde, usado para cobrir áreas diversas, tem sido confundido com outros termos de uso frequente. Assim, devo fazer uma distinção que considero muito importante, que é a distinção entre Psicologia da Saúde e Psicologia Clínica. Essa discussão já foi também estabelecida em outros artigos (Castro \& Bornholdt, 2004; Kerbauy, 2002; Miyazaki, Domingos, Valério, Santos \& Rosa, 2002; Yamamoto \& Cunha, 1998; Yamamoto, Trindade \& Oliveira, 2002;), mas é necessário enfatizá-la. Psicologia da Saúde NÃO É a Psicologia Clínica aplicada ao ambiente da saúde. Psicologia Clínica é uma prática mais antiga, existente desde os primórdios da Psicologia, e pode ser entendida, numa conceituação livre, como a aplicação dos conhecimentos do campo da Psicologia à solução de problemas pessoais ou grupais. Em geral, envolve o atendimento ou tratamento psicoterápico de um indivíduo que padece de algum distúrbio comportamental e/ou emocional. Por outro lado, os indivíduos atendidos em Psicologia da Saúde não precisam obrigatoriamente ter um distúrbio psicológico. O que distingue o campo da Psicologia da Saúde de outros campos da Psicologia é o fato que os indivíduos aqui atendidos têm, em geral, um problema ligado à sua saúde física, de diversas ordens ou gravidades possíveis. Usualmente, trata-se de um indivíduo que tem um problema orgânico relacionado a aspectos comportamentais ou emocionais, podendo tanto o problema orgânico quanto os aspectos comportamentais/ emocionais serem causa ou consequência da relação.

Não se pode simplesmente imaginar que um psicólogo que tenha uma boa formação clínica possa transferir suas habilidades e seu modus operandi da clínica para a área da saúde. Logicamente, existem alguns princípios e conceitos comuns a ambas as áreas, mas é fundamental que se estabeleça a diferença. Para um psicólogo ser um bom psicólogo da saúde, ele precisa conhecer bem o contexto onde vai trabalhar, seja um hospital, um ambulatório, um posto de saúde, uma comunidade ou o local de trabalho das pessoas. $\mathrm{O}$ ambiente, várias vezes, é determinante dos procedimentos que poderão ser utilizados e, indiscutivelmente, é determinante dos padrões comportamentais de adoecer, ficar saudável ou melhorar a qualidade de vida. Na clínica psicológica trabalha-se em uma situação protegida, para que a generalização para o ambiente natural depois ocorra. Na Psicologia da Saúde, em geral, trabalha-se no próprio contexto onde o comportamento ocorre. Há que se ter as habilidades peculiares a essa situação, sendo fundamental uma análise detalhada do ambiente, de fatores culturais, psicológicos e emocionais predisponentes à doença. Assim, um conhecimento de epidemiologia e fatores psicossociais de risco para doenças físicas se faz necessário para uma boa atuação profissional do psicólogo da saúde. Depois, há que se ter habilidades de relacionamento interpessoal acuradas para poder desenvolver o trabalho, que grande parte das vezes é multidisciplinar. Assim, também o domínio e a familiaridade com as demais áreas de conhecimento em saúde, como a Medicina, Enfermagem, Fisioterapia, Nutrição e outras, são fundamentais. Muitas vezes, o trabalho a ser feito com um paciente só pode ser feito se houver uma equipe atuando conjuntamente, onde cada um conhece e respeita o trabalho do outro, ao contrário da clínica, onde a particularidade da relação terapeuta-cliente é especial, a privacidade é sempre resguardada, o chamado setting ou ambiente e o clima do relacionamento são essenciais. Não se discute aqui se uma especialidade seria maior ou melhor do que a outra, mas sim que ambas são muito diferentes entre si, com peculiaridades próprias.

Outra questão de uso de termos que acho importante abordar neste artigo é a do uso do termo Psicologia Hospitalar. Vários fazem confusão entre Psicologia da Saúde e Psicologia Hospitalar. Não se trata do mesmo conceito. Psicologia Hospitalar é uma área importante dentro da Psicologia da Saúde, com necessidade de uma intervenção precisa e adequada em um ambiente acostumado a raciocinar com base em evidências (Gorayeb, 2001; Gorayeb \& Guerrelhas, 2003). Mas, como bem colocam Castro e Bornholdt (2004), aqui há uma confusão de termos tipicamente brasileira. Psicologia da Saúde é um termo que tem uma conotação mais ampla, incluindo a Psicologia que se pratica em hospitais. Infelizmente, os termos são confundidos entre si. O próprio Conselho Federal de Psicologia contribui para essa confusão ao instituir uma especialização em Psicologia Hospitalar e não ter uma especialização em Psicologia da Saúde. Parte da origem da questão é que uma grande parcela dos psicólogos que começaram a trabalhar em Psicologia da Saúde o fez em ambientes hospitalares. Provavelmente, há mais psicólogos 
trabalhando em hospitais do que nos outros níveis de atenção à saúde. Mas não se pode definir uma área de conhecimento pelo seu local de atuação, bem como não se deve tomar a parte pelo todo.

Pela sua abrangência, gosto da definição de Psicologia da Saúde apresentada por Matarazzo (1980):

Um conjunto de contribuições educacionais, cientificas e profissionais da disciplina da Psicologia para a promoção $e$ manutenção da saúde, a prevenção e tratamento de doenças, a identificação da etiologia e diagnóstico dos correlatos de saúde, doença e funções relacionadas, e a análise e aprimoramento do sistema e regulamentação da saúde. (p. 815)

Evoluindo a partir dessa definição, ficam facilmente distintos em Psicologia da Saúde os três níveis essenciais de intervenção preconizados pelo Sistema Único de Saúde (SUS) (Ministério da Saúde, 2010), criado em 1988 pela Constituição Federal Brasileira, para ser o sistema de saúde de toda a população. São muito conhecidos os três níveis básicos de atenção à saúde: a atenção primária, que se dá nos postos de saúde com o intuito de tratar alterações do estado de saúde mais simples; a atenção secundária, que se dá já em um nível de especialidade, em geral em situação ambulatorial; e a atenção terciária, que é prestada em hospitais. O nível de atenção terciária em Psicologia poderia ser o que se convencionou no Brasil chamar de Psicologia Hospitalar. Poder-se-ia, hoje, com a sofisticação dos níveis de atenção que se presta à saúde, falar até em uma atenção quaternária, em centros ultrassofisticados e especializados em tratamentos de problemas específicos ou em ambientes como uma Unidade de Terapia Intensiva.

Dada sua relevância para a saúde pública, dentro da área de Psicologia da Saúde também se deve destacar a importância da Promoção de Saúde, que não se faz só em hospitais ou ambulatórios, mas que pode se feita em qualquer contexto, tais como locais de trabalho, lares, escolas, Núcleos de Saúde da Família ou conjuntos habitacionais. São ações que um psicólogo da saúde, só ou em parceria com outros profissionais da saúde, executa para melhorar a qualidade de vida de uma parcela da população que se encontra saudável, mas que pode melhorar sua condição de existência. Ainda é pequena a produção nessa área no Brasil, apesar da mesma ser muito importante, não só por prevenir a ocorrência de doenças, promover melhoria na qualidade de vida das pessoas, mas também por levar a uma redução de custos financeiros no sistema de saúde. Alguns exemplos de atuação nessa área podem ser encontrados com o uso do Programa de Ensino de Habilidades de Vida da Organização Mundial da Saúde (Gorayeb, Netto \& Bugliani, 2003; Murta \& cols., 2010). Dentro dessa área, como em toda a atenção primária, devese destacar a criação dos Programas de Saúde da Família (Ministério da Saúde, 1996), que são assim definidos em sua página eletrônica:

A Saúde da Família é entendida como uma estratégia de reorientação do modelo assistencial, operacionalizada mediante a implantação de equipes multiprofissionais em unidades básicas de saúde. Estas equipes são responsáveis pelo acompanhamento de um número definido de famílias, localizadas em uma área geográfica delimitada. As equipes atuam com ações de promoção da saúde, prevenção, recuperação, reabilitação de doenças e agravos mais frequentes, e na manutenção da saúde desta comunidade.

Por essa própria definição, onde fatores de ordem comportamental, emocional e cognitiva certamente têm sua influência, pode-se dizer que, mesmo sem fazer parte da equipe básica preconizada, o psicólogo tem um importante papel. Poucas são as publicações de psicólogos na área, que deve ser caracterizada por ações multiprofissionais e interdisciplinares (Neckel, Seemann, Eidt, Rabuske \& Crepaldi, 2009), sendo que aqui também um incremento de ações e publicações se faz necessário.

\section{Perspectivas para o Futuro}

Para favorecer o desenvolvimento futuro da Psicologia da Saúde no Brasil, julgo que algumas condições se fazem necessárias. Primeiramente, julgo que a disciplina deveria ser sempre ministrada em cursos de graduação, com ênfase nas possibilidades de atuação e pesquisa em diversos níveis de atenção à saúde. Depois, haveria que se estimular a existência de programas de formação ao nível pós-graduado, seja em especializações (e. g., Residências), seja em programas de pós-graduação stricto sensu. Também, a área depende muito de instrumentos adequados para mensuração dos diversos aspectos de comportamentos, sentimentos e cognições dos indivíduos, para analisar a eficácia das intervenções. Boa parte dos instrumentos utilizados em Psicologia da Saúde ainda é de origem estrangeira, alguns com uma boa tradução, adaptação, validação e normatização, como as Escalas de Beck para Depressão e Ansiedade (Cunha, 2001); outros, apesar de seus estudos de adaptação terem sido executados com amostra pequena, têm se mostrado úteis e sido largamente utilizados (Botega, Zomignani \& Wab, 1995). Há também exemplos de produção nacional de instrumentos que são úteis na área de Psicologia da Saúde (Lipp, 2000). Um instrumento criado pela Organização Mundial da Saúde para avaliar qualidade de vida, o WHOQOL-Bref, adaptado para o Brasil por Fleck e cols. (2000), tem também sido muito utilizado, especialmente em sua versão abreviada. Todavia, sejam os adequados estudos de tradução, adaptação, validação e normatização, sejam as criações nacionais, o leque de instrumentos disponíveis para pesquisa na área de Psicologia da Saúde é ainda incompleto e insuficiente. Faz-se necessária a criação de novos instrumentos para medir os diversos aspectos dos comportamentos, emoções ou cognições que ocorrem nos cuidados com a saúde. Laboratórios de Psicometria, como o do Prof. Luiz Pasquali ${ }^{5}$, na Universidade de Brasília (UnB), estão crescendo em produção. Na ANPEPP, também existem grupos organizados de pesquisadores trabalhando nesse domínio. Tudo isto pode ajudar o desenvolvimento de instrumentos de medida mais específicos para a área da saúde.

5 Homenageado pela Universidade de Brasília com o título de Professor Emérito (ver o texto produzido pelos professores do Departamento de Psicologia Social e do Trabalho/UnB, 2007). 
Nessa análise de condições necessárias para o desenvolvimento da área, há que se destacar o papel das sociedades científicas. Eu, pessoalmente, lamento que no Brasil haja uma dissociação entre o Conselho Profissional e essas sociedades. Em outros países, como o Canadá, onde essas entidades são interligadas, há um favorecimento maior do desenvolvimento da profissão, com o conhecimento científico servindo como suporte e apoio para as decisões de ordem profissional, como por exemplo, na elaboração do Código de Ética. Mas, essa é uma evolução que ainda se vai ter que esperar amadurecimento para que ocorra no Brasil, dados os interesses políticos e pessoais envolvidos na questão. Dentre as sociedades científicas que, a meu ver, mais têm contribuído para o desenvolvimento da Psicologia da Saúde no Brasil, destaco a SBP, com suas reuniões anuais ocorrendo ininterruptamente há 40 anos. Também, com sua origem há 19 anos, a ABPMC é outra sociedade científica onde o conhecimento baseado em evidências tem encontrado grande apoio para sua divulgação. Além destas, a Sociedade Brasileira de Psicologia Hospitalar tem dado sua contribuição para a área.

Deve-se, aqui, destacar o papel extremamente importante para o desenvolvimento da pesquisa e do ensino em Psicologia da Saúde no Brasil que a ANPEPP tem. Ali, Raquel Kerbauy coordenou durante vários anos um grupo de trabalho em Psicologia da Saúde que ajudou a divulgar e melhor estabelecer a área no campo da Psicologia. Posteriormente, tive o orgulho de coordenar um grupo de trabalho na ANPEPP intitulado "Psicologia da Saúde em Instituições e na Comunidade", com a participação de pesquisadores de diversas universidades e estados brasileiros, incluindo, além de professores já citados, Tânia Rudnick, da ULBRA - Canoas, Suely Guimarães e Eliane Fleury Seidl, da UnB. Esses pesquisadores trabalham na área de Psicologia da Saúde, procuram ter uma produção conjunta e estimulam seus alunos e outros pesquisadores com suas palestras, conferências e publicações. Além desse grupo, que conheço com mais detalhes, há outros importantes grupos de pesquisadores na área de Psicologia da Saúde na ANPEPP.

Enfim, resta dizer que com o crescimento de publicações, que certamente ocorrerá, com uma adequada inserção do conceito de Psicologia da Saúde no conteúdo básico de ensino de graduação e com a oferta de novas oportunidades de formação pós-graduada, a área de Psicologia da Saúde deve vir a crescer ainda mais no Brasil, trazendo sua contribuição para o bem-estar das pessoas. A tarefa não é fácil, os obstáculos não são poucos, haverá resistências pelo caminho, mas temos que ser otimistas, com o apoio de nosso próprio conhecimento. Para isso, gostaria, ao finalizar, de repetir uma frase que Fred Keller usou para encerrar um curso de graduação, muitos anos atrás, na Columbia University, relembrada e transcrita por um de seus alunos da época, num artigo de homenagem póstuma ao grande mestre (Cook, 1996):

The world is an awful place. People in it know much and accomplish much, but spread suffering among each other and seem unable to avoid this. They even know something of this fact, but that seems not to be enough. We need the kind of knowledge that will actually change how people do things, so that the amount of suffering might diminish. I believe that a science of behavior can make a contribution to that end. (p. 70)

Com essa mensagem deixo também meus agradecimentos à revista Psicologia: Teoria e Pesquisa, pela oportunidade de expressar minha opinião em um tema tão relevante para a saúde da população e para o futuro da Psicologia brasileira.

\section{Referências}

Assis, F. R. P., Delitti, M., \& Lima, M. V. O. (2005). Homenagem a Maria Amélia Matos. Revista Brasileira de Terapia Comportamental e Cognitiva, III, 1-3.

Botega, N. J., Zomignani, M., \& Wab, P. (1995). Transtornos do humor em uma enfermaria de clínica médica e validação de escala de medida (HAD) de ansiedade e depressão. Revista de Saúde Pública (São Paulo), 29, 355-363.

Bueno, J. L. O. (2006). Homenagem a sócio honorário: Luiz Marcellino de Oliveira. Temas em Psicologia, 14, 13-14. Retirado em 01/08/2010, de http://www.sbponline.org.br/revista2/vol14n1/ PDF/v14n01a03.pdf

Castro, E. K., \& Bornholdt, E. (2004). Psicologia da Saúde x Psicologia Hospitalar: definições e possibilidades de inserção profissional. Psicologia Ciência e Profissão, 24, 48-57.

Conselho Federal de Psicologia (2005). Homenageado: Mathilde Neder. Psicologia: Ciência e Profissão, 25, 332.

Cook, D. A. (1996). Reminiscences. Fred S. Keller: An appreciation. Behavior and Social Issues, 6, 61-71.

Crepaldi, M. A., Linhares, M. B. M., \& Perosa, G. B. (2006). Temas em Psicologia Pediátrica. São Paulo: Casa do Psicólogo.

Cunha, J. A. (2001). Manual da versão em português das Escalas Beck. São Paulo: Casa do Psicólogo.

Cunha, R. N. (2007). João Claudio Todorov: Planejador de contingências para o aprender a aprender e para o aprender a ensinar. Psicologia, Teoria e Pesquisa, 23, 53-55.

Departamento de Processos Psicológicos Básicos/UnB (2007). Professor Emérito João Claudio Todorov. Psicologia: Teoria e Pesquisa, 23, 51-52.

Departamento de Psicologia Escolar e do Desenvolvimento/ UnB (2007). Professora Emérita Thereza Pontual de Lemos Mettel. Psicologia: Teoria e Pesquisa, 23, 125-129.

Departamento de Psicologia Social e do Trabalho/UnB (2007). Professor Emérito Luiz Pasquali. Psicologia: Teoria e Pesquisa, 23, 97-98.

Dessen, M. A. (2006). Homenagem à sócia honorária: Maria Clotilde Therezinha Rossetti Ferreira. Temas em Psicologia, 14, 15-16.

Feitosa, M. A. G. (2007). Doutora Honoris Causa Carolina Martuscelli Bori (1924-2004). Psicologia: Teoria e Pesquisa, $23,25-28$

6 "O mundo é um lugar terrível. As pessoas sabem muito e realizam muito, mas disseminam sofrimento entre si e parecem incapazes de evitar isto. Elas até sabem algo sobre este fato, mas isto não parece ser suficiente. Nós precisamos de um tipo de conhecimento que venha realmente mudar como as pessoas fazem as coisas, de forma que a quantidade de sofrimento possa diminuir. Eu acredito que uma ciência do comportamento possa dar uma contribuição neste sentido". (tradução livre do autor deste artigo) 
Ferrari, E. A. M. (2008). The pioneer contribution of Luiz Marcellino de Oliveira to experimental analysis of behavior and psychobiological investigation in Brazil. Psychology \& Neuroscience, 1, 99-102. Retirado em 01/08/2010, de http://www. psycneuro.org/index.php/psycneuro/article/view/33/126.

Fleck, M. P. A., Louzada, S., Xavier, M., Chachamovich, E., Vieira, G., Santos, L., \& Pinzon, V. (2000). Aplicação da versão em português do instrumento WHOQOL-bref. Revista de Saúde Pública, 34, 178-183.

Gorayeb, R. (1983). O papel profissional da ação do psicólogo num Hospital Geral. Cadernos Prohasa, 1, 122-132.

Gorayeb, R. (1984). A participação do psicólogo na tarefa médica. Boletins de Psiquiatria, 17, 118-122.

Gorayeb, R. (1985). Residência em psicologia clínica. Cadernos FUNDAP, 5, 66-68.

Gorayeb, R. (1990). História da Sociedade de Psicologia de Ribeirão Preto. Ciência e Cultura, 42, 827-831. Retirado em 20/08/2010, de http://www.sbponline.org.br/historico.php.

Gorayeb, R. (2001). A prática da Psicologia Hospitalar. Em M. L. Marinho \& V. E. Caballo (Orgs.), Psicologia Clínica e da Saúde (pp. 263-278). Londrina: UEL-APICSA. 15,8 .

Gorayeb, R. (2005). Carolina Bori, Viva para sempre!. Paidéia,

Gorayeb, R., \& Guerrelhas, F. (2003). Sistematização da prática psicológica em ambientes médicos. Revista Brasileira de Terapia Comportamental e Cognitiva, 5, 11-19.

Gorayeb, R., Netto, J. R. C., \& Bugliani, M. A. P. (2003). Promoção de saúde na adolescência: experiência com Programas de Ensino de Habilidades de Vida. Em Z. A. Trindade \& A. N. A. Andrade (Orgs.), Psicologia e saúde: um campo em construção (pp. 89-100). Casa do Psicólogo: São Paulo.

Guedes, M. C. (2005). Relembrando Carolina Bori, Paidéia, 15, 9-10.

Ismael, S. M. C. (2005). Temas de prevenção, ensino e pesquisa que permeiam o contexto hospitalar. Casa do Psicólogo: São Paulo.

Jurberg, C. (2010). Frederico Guilherme Graeff: uma prosa em verso cientifico. Retirado em 20/08/2010, de http://www.sbnec.org. $\mathrm{br} /$ site/index.php?page=frederico-graeff.

Keller, F. S. (1966). A personal course in psychology. Em R. Ulrich, T. Stachnick \& J. Mabry (Eds.), The control of behavior (pp. 91-93). Glenview: Scott, Foresman.

Keller, F. S. (1968). “Good-bye, Teacher...”. Journal of Applied Behavior Analysis, 1, 79-89.

Kerbauy, R. R. (1999). Comportamento e saúde: explorando alternativas. Santo André: ARBytes.

Kerbauy, R. R. (2002). Comportamento e saúde: doenças e desafios. Psicologia USP, 13, 11-28.

Kerbauy, R. R. (2004) A presença de Carolina Martuscelli Bori na Psicologia. Revista Brasileira de Terapia Comportamental e Cognitiva, VI, 159-164.

Lettner, H. W., \& Rangé, B. P. (1988). Manual de psicoterapia comportamental. São Paulo: Manole.
Lipp, M. N. (2000). Manual do Inventário de Sintomas de Stress para Adultos de Lipp (ISSL). São Paulo: Casa do Psicólogo.

Matarazzo, J. D. (1980). Behavioral health and behavioral medicine: Frontiers for a new health psychology. American Psychologist, 35, 807-817.

Matos, M. A., 1998, Contingências para a Análise Comportamental no Brasil. Psicologia USP, 9, 89-100.

Ministério da Educação (2007). Portaria Interministerial MEC/ MS no. 698. Retirado em 20/08/2010, de http://portal.mec.gov.br/ sesu/arquivos/pdf/residencia/portaria_698_07.pdf.

Ministério da Saúde (1996). Saúde da Família: uma estratégia de organização dos serviços de saúde. Brasília: Ministério da Saúde.

Ministério da Saúde (2010). Sobre o SUS. Retirado em 20/08/2010, de http://portal.saude.gov.br/portal/saude/default.cfm.

Miyazaki, M. C. O. S., Domingos, N. A. M., \& Valério, N. I. (2006). Psicologia da Saúde: pesquisa e prática. São José do Rio Preto: THS/Arantes.

Miyazaki, M. C. O. S., Domingos, N., Valério, N., Santos, A. R. R., \& Rosa, L. T. B. (2002). Psicologia da Saúde: extensão de serviços à comunidade, ensino e pesquisa. Psicologia USP, 13, 29-53.

Murta, S. G., Borges, F. A., Ribeiro, D. C., Rocha, E. P., Menezes, J. C. L., \& Prado, M. M. (2010). Prevenção primária em saúde na adolescência: avaliação de um programa de habilidades de vida. Estudos de Psicologia (Natal), 14, 181-189.

Neckel, G. L., Seemann, G., Eidt, H. B., Rabuske, M. M., \& Crepaldi, M. A. (2009). Desafios para a ação interdisciplinar na atenção básica: implicações relativas à composição das equipes de saúde da família. Ciência \& Saúde Coletiva, 14, 1463-1472.

Neder, M. (1995). O psicólogo no hospital: o início das atividades psicológicas no HCFMUSP. Mundo Saúde, 27, 326-336.

Pires, A. C. T., \& Braga, T. M. S (2009). O psicólogo na saúde pública: formação e inserção profissional. Temas em Psicologia, 17, 151-162. Retirado em 20/08/2010, de http://www.sbponline. org.br/revista2/vol17n1/v17n1a13ab.htm.

Rozestraten, R. J. A. (1988). Histórico da Sociedade Brasileira de Psicologia: os primórdios da SBP. Retirado em 20/08/2010, de http://www.sbponline.org.br/historico.php.

Sebastiani, R. W. (2003). Psicología de la salud en Brasil: 50 años de historia. Suma Psicológica, 10, 25-42.

Straub, R. O. (2005). Psicologia da Saúde. Porto Alegre: Artmed.

Tomanari, G. Y. (2005). Notícia. Maria Amélia Matos (19392005): generosidade, competência, liderança. Psicologia: Teoria e Pesquisa, 21, 255-256.

Yamamoto, O. H., \& Cunha, I. M. F. F. O. (1998). O psicólogo em hospitais de Natal: uma caracterização preliminar. Psicologia: Reflexão e Crítica, 11, 345-362.

Yamamoto, O. H., Trindade, L. C. B. O., \& Oliveira, I. F. (2002). O psicólogo em hospitais no Rio Grande do Norte. Psicologia USP, 13, 217-246. 